\title{
Monocytic MDSC as a source of immunosuppressive cytokines in chronic lymphocytic leukemia (CLL) microenvironment
}

\author{
Wioleta Kowalska, Agnieszka Bojarska-Junak
}

Chair and Department of Clinical Immunology, Medical University of Lublin, Poland

\begin{abstract}
Introduction. Myeloid derived suppressor cells (MDSCs) are one of the major components of the tumor microenvironment. The accumulation of MDSCs has been demonstrated in many types of human solid tumors. However, the relevance of this heterogeneous population in hematopoietic malignancies has only recently gained stronger attention. MDSCs are a phenotypically and functionally heterogeneous group of cells. The results of recent studies indicate that the immune dysregulation in chronic lymphocytic leukemia (CLL) affects a monocytic MDSC (M-MDSC) subpopulation. This study aimed to analyze the frequency of M-MDSCs with intracellular IL-10 and TGF- $\beta 1$ expression in newly diagnosed CLL patients. We investigated the potential role of M-MDSCs in CLL by analyzing the level of IL- 10 and TGF- $\beta 1$ expression in circulating M-MDSCs in correlation with clinical and laboratory parameters characterizing disease activity and patients' immune status.

Material and methods. Seventy CLL patients and 17 age-matched healthy volunteers were included in this study. Flow cytometric detection of Mo-MDSCs (CD14+ ${ }^{+}$D $11 b^{+}$CD15-HLA-DR $\left.{ }^{- \text {llow }}\right)$ with intracellular IL-10 and TGF- $\beta 1$ expression was done.

Results. We found a significantly higher median percentage of M-MDSC with IL-10 or TGF- $\beta 1$ expression in CLL patients than in healthy volunteers. The percentage of M-MDSC with intracellular IL-10 or TGF- $\beta 1$ expression was significantly lower in CLL patients at stage 0 as compared to the stages I/II and III/IV according to Rai stages. The percentage of M-MDSC with intracellular TGF- $\beta 1$ expression was significantly higher in ZAP-70-positive and CD38-positive patients compared with ZAP-70-negative and group of CD38-negative ones. There was also a significantly higher percentage of M-MDSC positive for intracellular TGF- $\beta$ expression in patients carrying the $11 \mathrm{q} 22.3$ and/or the $17 \mathrm{p} 13.1$ deletion than in patients without these genetic aberrations. The percentage of M-MDSC IL-10-positive and M-MDSC TGF- $\beta$ 1-positive measured at the time of diagnosis was higher in patients requiring therapy as compared to patients without treatment during the observation period. Conclusion. In conclusion, we have shown that an increased percentage of M-MDSC cells producing IL-10 and TGF- $\beta 1$ in CLL patients may be associated with the suppression of the immune response against CLL. It can be assumed that the increased percentage of M-MDSC with an intracellular expression of IL-10 and TGF- $\beta 1$ may be used in the future as the factor defining the group of patients with shorter time to onset of treatment. (Folia Histochemica et Cytobiologica 2020, Vol. 58, No. 1, 25-36)
\end{abstract}

Key words: M-MDSC; IL-10; TGF- $\beta$; chronic lymphocytic leukemia; flow cytometry; genetic analysis

Correspondence address: Agnieszka Bojarska-Junak, $\mathrm{PhD}$ Chair and Department of Clinical Immunology

Medical University of Lublin, Chodzki 4a, 20-093 Lublin, Poland

tel. +4881 4486420; fax +48 814486421

e-mail: abojarskajunak@gmail.com

\section{Introduction}

Chronic lymphocytic leukemia (CLL) is characterized by an abnormal expansion of mature CD5 + B cells in the bone marrow and their accumulation in blood and secondary lymphoid organs [1]. The neoplastic B cells in CLL patients are dependent on interactions with their microenvironment. CLL cells fluctuate together with a microenvironment, which supports leukemia 
cell survival and determines disease progression in CLL [2-5]. MDSCs (myeloid- derived suppressor cells) are one of the major components of the tumor microenvironment. The accumulation of MDSCs has been demonstrated in many types of human solid tumors [6]. However, the relevance of this heterogeneous population in hematopoietic malignancies has only recently gained stronger attention [7-10]. MDSCs are a phenotypically and functionally heterogeneous group of cells. In human there are two major types of MDSCs: monocytic-MDSC (M-MDSC; expressing CD14) and polymorphonuclear MDSC (PMN-MDSC; expressing CD15) [5, 11, 12]. Both subpopulations of MDSCs express the myeloid marker CD33. Unlike monocytes, MDSCs are characterized by the lack or low expression of HLA-DR [5]. M-MDSCs produce high amounts of immunosuppressive cytokines, such as IL-10 and TGF- $\beta$ [13].

In CLL, malignant B lymphocytes, serve an important role in the immune response, so their interactions with other immune cells are more complex than observed in solid tumors. The results of recent studies indicate that the immune dysregulation in CLL also affects MDSCs [14, 15]. Jitschin et al. [16] observed the accumulation of M-MDSCs (defined as CD14 ${ }^{+} \mathrm{H}_{-}$ LA-DR ${ }^{\text {lo }}$ cells) in peripheral blood of CLL patients. Probably, some cytokines, such IL-10, contribute to the recruitment and accumulation of MDSCs in CLL microenvironment $[17,18]$. In addition, it has been shown that CLL cells themselves constitutively produce immunosuppressive IL-10 [19]. The course of CLL is accompanied by a state of immunosuppression. The leukemic B cells achieve this mainly through the involvement of regulatory $\mathrm{T}$ (Treg) cells. In turn, MDSCs release IL-10 and TGF- $\beta$ to exert their suppressive function by Tregs $[8,20]$. The origin of M-MDSC is unknown. Some scientists believe that M-MDSCs are derived from emergency myelopoiesis that can occur during cancer development [12, 21, 22]. Other theories suggest that M-MDSCs are formed in the pathways of reprogramming monocytes [23]. Probably, this process is connected with some cytokine milieu (e.g. IL-10) [22, 24].

In a recent study, we found the accumulation of intermediate and non-classical monocytes in CLL patients. The non-classical monocytes constituted the majority of cells lacking HLA-DR expression. Moreover, non-classical monocytes presented low CD163 expression [25]. HLA-DR ${ }^{\text {low/ }} \mathrm{CD} 163^{\text {low/ }}$ phenotype is also characteristic for M-MDSC [23].

This study aimed to analyze the frequency of M-MDSCs with intracellular IL-10 and TGF- $\beta 1$ expression in newly diagnosed CLL patients. We investigated the potential role of M-MDSCs in CLL by analyzing the level of IL-10 and TGF- $\beta 1$ expression in circulating M-MDSCs in correlation with clinical and laboratory parameters characterizing disease activity and patients' immune status.

\section{Material and methods}

Patients and samples. The study group comprised 70 patients with CLL diagnosis, which was based on criteria from the International Workshop on Chronic Lymphocytic Leukemia (IWCLL) [26]. All subjects were newly diagnosed. Peripheral blood (PB) samples were collected at the time of diagnosis and prior to any anticancer therapy. CLL patients were recruited between January 2016 and June 2019 in the Department of Hematooncology and Bone Marrow Transplantation of the Medical University of Lublin (Lublin, Poland). Clinical stage was determined according to the Rai classification system [27]. Thirty-seven patients were Stage 0,11 patients were Stage I, 12 patients were Stage II, 7 patients were Stage III and 3 patients were Stage IV. The CLL group was further divided into three risk groups: Low risk (stage 0), intermediate-risk (stage I or II), and high-risk (stage III or IV). Characteristics of patients at the time of diagnosis are summarized in Table 1. Control PB samples were obtained from 17 healthy volunteers (HVs; 8 females and 9 males, aged from 35-74 years, median 56 years).

Peripheral blood (PB) samples were collected into EDTA-coated tubes and immediately processed. Peripheral blood mononuclear cells (PBMCs) were separated by density gradient centrifugation on Gradisol L (Aqua-Med, Lodz, Poland) for $25 \mathrm{~min}$. at $400 \mathrm{~g}$ at room temperature (RT). Interphase cells were removed, washed twice and resuspended in phosphate-buffered saline (PBS).

Ethics statement. This study was approved by the Ethics Committee of the Medical University of Lublin (No. KE0254/107/2013 and KE-0254/49/2016). Written informed consent was obtained from all patients with respect to the use of their blood for scientific purposes.

Detection of M-MDSC and analysis of intracellular IL-10 or TGF- $\beta 1$ expression. Flow cytometry analysis of M-MDSC (defined as CD14+CD11b+CD15-HLA-DR-/low cells) was performed on PBMCs. The samples were stained with combination of fluorescent-labelled monoclonal antibodies (MoAbs): mouse anti-human 14 FITC (Clone M $\varphi$ P9), mouse anti-human CD11b V450 (Clone ICRF44), mouse anti-human HLA-DR PE-Cy7 (Clone L243) and CD15 APC (Clone HI98) (BD Biosciences, Franklin Lakes, NJ, USA). Cells were incubated for $20 \mathrm{~min}$ at RT. Following membrane staining, cells were fixed with Cytofix/Cytoperm and permeabilized with Perm/Wash buffer (BD Biosciences) according to the manufacturer's protocol. Cells were then intracellularly stained (20 min at RT) with PE anti-human 
Table 1. Characteristics of the patients at CLL diagnosis

\begin{tabular}{|c|c|}
\hline Features & No. patients $(\%)$ \\
\hline \multicolumn{2}{|l|}{ Sex } \\
\hline Female $(\%)$ & $33(47.1)$ \\
\hline Male $(\%)$ & $37(52.9)$ \\
\hline \multicolumn{2}{|l|}{ Rai Stage } \\
\hline $0(\%)$ & $37(52.9)$ \\
\hline $\mathrm{I}(\%)$ & $11(15.7)$ \\
\hline II $(\%)$ & $12(17.1)$ \\
\hline III $(\%)$ & $7(10.0)$ \\
\hline IV $(\%)$ & $3(4.3)$ \\
\hline \multicolumn{2}{|l|}{ ZAP-70 (cut-off 20\%) $)^{\mathrm{a}}$} \\
\hline Positive (\%) & $24(34.3)$ \\
\hline Negative $(\%)$ & $46(65.7)$ \\
\hline \multicolumn{2}{|l|}{ CD38 (cut-off 20\%) } \\
\hline Positive (\%) & $25(35.7)$ \\
\hline Negative (\%) & $45(64.3)$ \\
\hline \multicolumn{2}{|l|}{ Cytogenetic abnormalities } \\
\hline $\operatorname{del}(17 \mathrm{p} 13.1)(\%)$ & $1(1.4)$ \\
\hline $\operatorname{del}(11 \mathrm{q} 22.3)(\%)$ & $9(12.9)$ \\
\hline Without del(17p13.1) and del(11q22.3) (\%) & $60(85.7)$ \\
\hline Patients requiring therapy & $17(24.3)$ \\
\hline \multirow[t]{2}{*}{ Untreated patients } & $53(74.7)$ \\
\hline & median (range) \\
\hline Age at diagnosis (years) & $65(46-85)$ \\
\hline WBC count $[\mathrm{G} / \mathrm{L}]$ & $21.92(10.11-290.46)$ \\
\hline Lymphocyte count [G/L] & $16.57(5.21-284.9)$ \\
\hline$\beta_{2} \mathrm{M}[\mathrm{mg} / \mathrm{dl}]$ & $2.42(1.35-5.39)$ \\
\hline LDH [IU/1] & $362.0(178.0-492.0)$ \\
\hline Hemoglobin $[\mathrm{g} / \mathrm{dl}]$ & $13.95(8.7-16.8)$ \\
\hline Platelets $[\mathrm{G} / \mathrm{L}]$ & $187.0(23.0-414.0)$ \\
\hline$\% \mathrm{CD} 19^{+} / \mathrm{CD}^{+} / \mathrm{ZAP}-70^{+}$cells $^{\mathrm{a}}$ & $18.0(0.2-50.0)$ \\
\hline$\% \mathrm{CD} 19^{+} / \mathrm{CD}^{+} / \mathrm{CD} 38^{+}$cells $^{\mathrm{b}}$ & $16.1(0.02-88.7)$ \\
\hline
\end{tabular}

CLL — chronic lymphocytic leukemia; WBC — white blood cell; LDH lactate dehydrogenase; $\beta 2 \mathrm{M}-\beta 2$ microglobulin. ${ }^{\text {aPatients with ZAP-70 }}$ expression lower or higher than $20 \%$ were classified as ZAP-70 negative or positive, respectively. ${ }^{\mathrm{b}}$ Patients with CD38 expression lower or higher than $30 \%$ were classified as CD38 negative or positive, respectively.

LAP (TGF- $\beta 1$ ) antibody (Clone TW4-2F8) or PE anti-human IL-10 antibody (Clone JES3-19F1) (BioLegend, San Diego, CA, USA).

Flow cytometry analysis. Samples were analyzed by flow cytometry directly following preparation. Data acquisition was performed on a FACSCanto II instrument with FACSDiva Software (BD Biosciences). For each analysis, 100,000 events were acquired and analyzed. Kaluza 2.1.1 (Beckman Coulter, Miami, FL, USA) was used for the data analysis. An acquisition gate was put on lymphocytes according to the forward scatter (FSC) and side scatter (SSC) properties. The gating strategy to identify M-MDSC with IL-10 or TGF- $\beta 1$ expression is shown in Figure $1 \mathrm{~A}-\mathrm{E}$. The results are expressed as the percentage of M-MDSC with intracellular IL-10 or TGF- $\beta 1$ expression. Furthermore, within M-MDSC population, IL-10 and TGF- $\beta 1$ cytokines were quantified regarding their mean fluorescence intensity (MFI). To establish the gating strategy, fluorescence minus one (FMO) control was used. The FMO control tube included all antibodies that were used for M-MDSC cell staining, except for the one (IL-10 PE or TGF- $\beta 1$ PE) that was measured.

Analysis of T regulatory cells (CD4+CD25+FoxP3+). Treg cells were evaluated via analysis of the surface expression of CD4 and CD25 antigens, as well as intracellular expression of FoxP3 by flow cytometry. Tregs were stained with $\mathrm{Hu}-$ man Treg Flow Kit (FOXP3 Alexa Fluor 488/CD4 PECy5/ /CD25 PE) (BioLegend) according to the manufacturer's instructions.

Analysis of CD38 and ZAP-70 expression in CLL cells. CLL cells were stained for CD38 antigen and ZAP-70 protein expression (as described previously [28]). Fresh PB samples were stained with FITC mouse anti-human CD19 (Clone SJ25C1), PE-Cy5 mouse anti-human CD5 (Clone UCHT2) and CD38 FITC (Clone HIT2) or anti-ZAP-70 $\mathrm{PE}$ (Clone 1E7.2) (BD Biosciences). A cut-off point for ZAP-70 positivity in leukemic cells was $\geq 20 \%$. Patients with CD38 expression lower or higher than $30 \%$ were classified as CD38 negative or positive, respectively.

I-FISH analysis. Detection of $\operatorname{del}(17 \mathrm{p} 13.1)$ and $\operatorname{del}(11 \mathrm{q} 22.3)$ was a part of the routine diagnostic practice. We used a previously described method [29].

Statistical analysis. The Kruskal-Wallis test with Dunn correction or U Mann-Whitney test were used for comparative analysis of the variables. The Spearman rank correlation coefficient was used in correlation tests. Differences were considered statistically significant with $\mathrm{p}$-value $\leq 0.05$. Statistical analysis was calculated with Statistica 13 PL (Statistica, Krakow, PL). Graphs were processed using GraphPad Prism version 5.

\section{Results}

The percentage of M-MDSC was significantly increased in patients with CLL in comparison to the HVs (median [IQR], 2.84\% [1.25-4.50\%] vs. 8.605 [6.82-11.12\%], $\mathrm{p}<0.001)$. Moreover, we found significantly higher median percentage of M-MDSC 

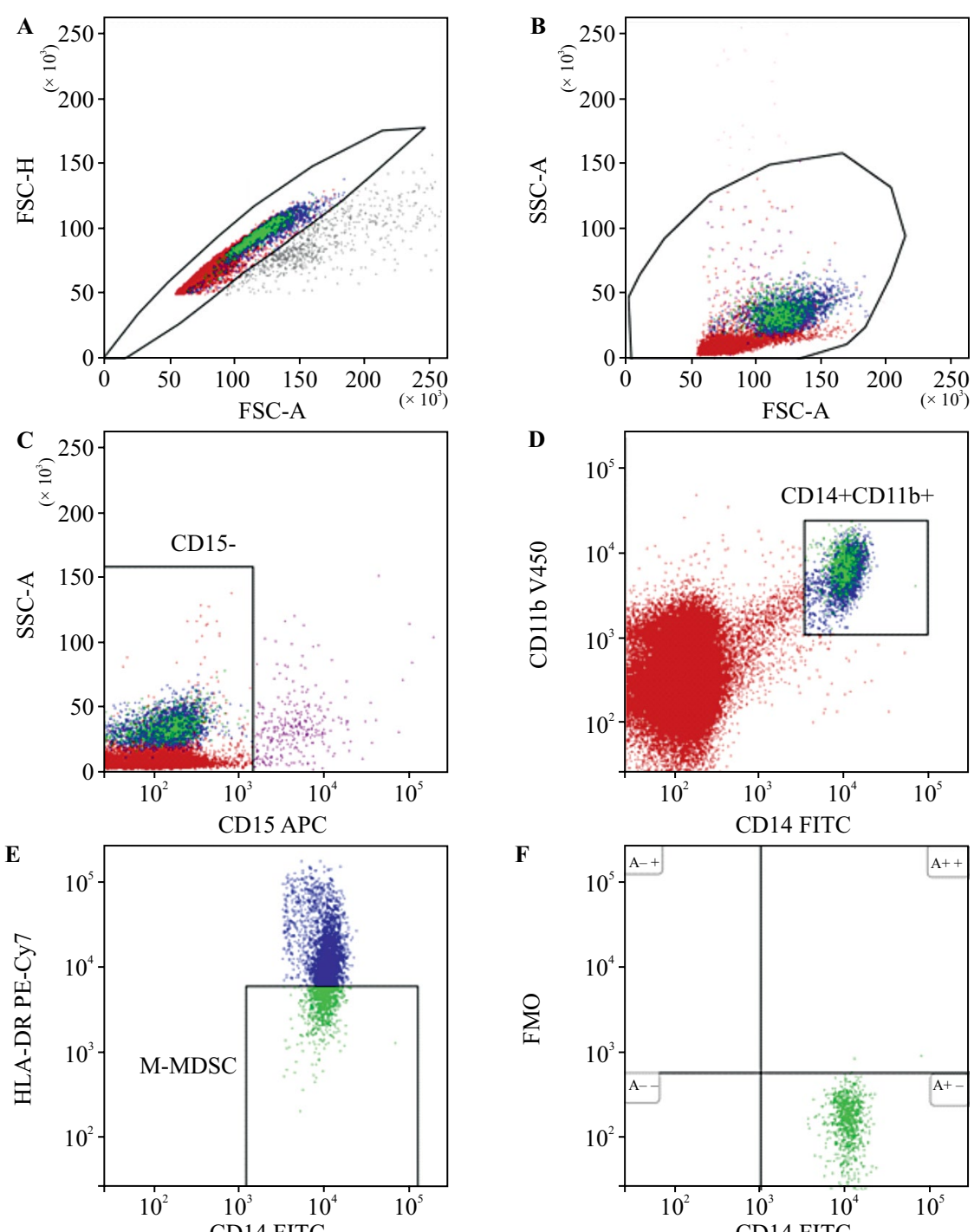

$\mathbf{F}$
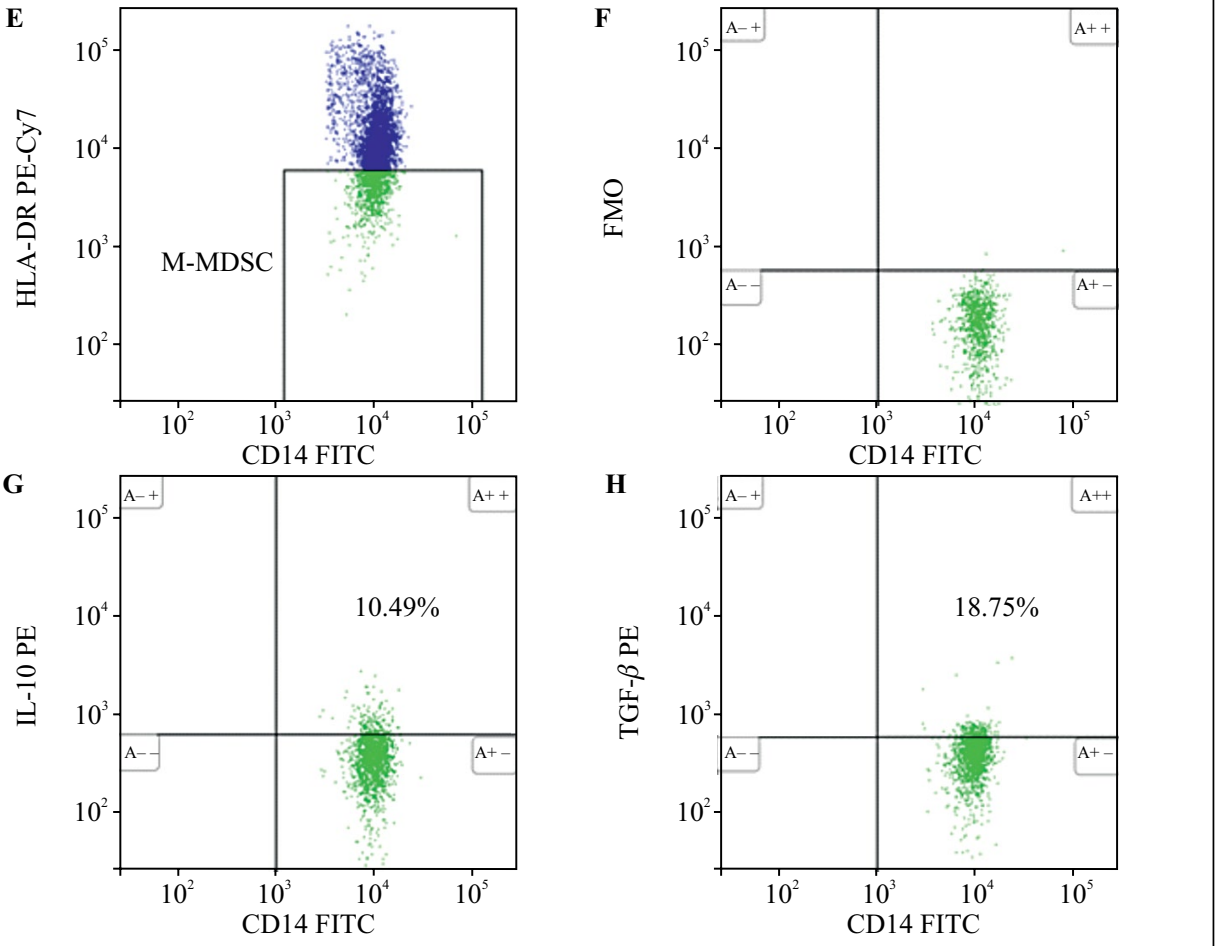

Figure 1. Representative dot plots illustrating the analytic method for the identification of M-MDSCs with the intracellular expression of IL-10 and TGF- $\beta 1$. A. FSC-A vs. FSC-H dot plot: doublets' discrimination. B. After gating a singlet (P1 region) mononuclear cells (PBMCs) were selected based on their SSC/FSC properties. C. CD15 APC vs. SSC-A dot plot. Discrimination of CD15- from CD15+ cells. D. Selected CD15- cells were analyzed for CD14 FITC and CD11b V450 staining. E. Selected CD14 ${ }^{+} \mathrm{CD} 11 \mathrm{~b}^{+}$cells were analyzed for HLA-DR PE-Cy7 expression. The dot plots (CD14 FITC vs. HLA-DR $\mathrm{PE}-\mathrm{Cy} 7$ ) indicate $\mathrm{CD} 14^{+} \mathrm{CD} 11 \mathrm{~b}^{+} \mathrm{CD} 15{ }^{-} \mathrm{HLA}_{-} \mathrm{DR}^{-/ \mathrm{low}}$ cells (M-MDSC). F. Dot plots indicating a FMO (Fluorescence minus one) control, which contains all fluorochromes in a panel except for IL-10 PE or TGF- $\beta$ PE. The FMO control identifies any spread of fluorochrome in an unlabeled channel and places the gates in the correct place. G, H. Selected CD14 ${ }^{+} \mathrm{CD}-$ $11 \mathrm{~b}^{+} \mathrm{CD} 15-\mathrm{HLA}_{-\mathrm{DR}}{ }^{-/ \mathrm{ow}}$ cells were analyzed for IL-10 PE (G) or TGF- $\beta$ PE staining (H). A++ quadrant in dot plot (G) shows the percentage of M-MDSCs IL-10-positive. A++ quadrant in dot plot (H) shows M-MDSCs with the intracellular expression of TGF- $\beta$. Data were analyzed using Kaluza 2.1.1 software. 


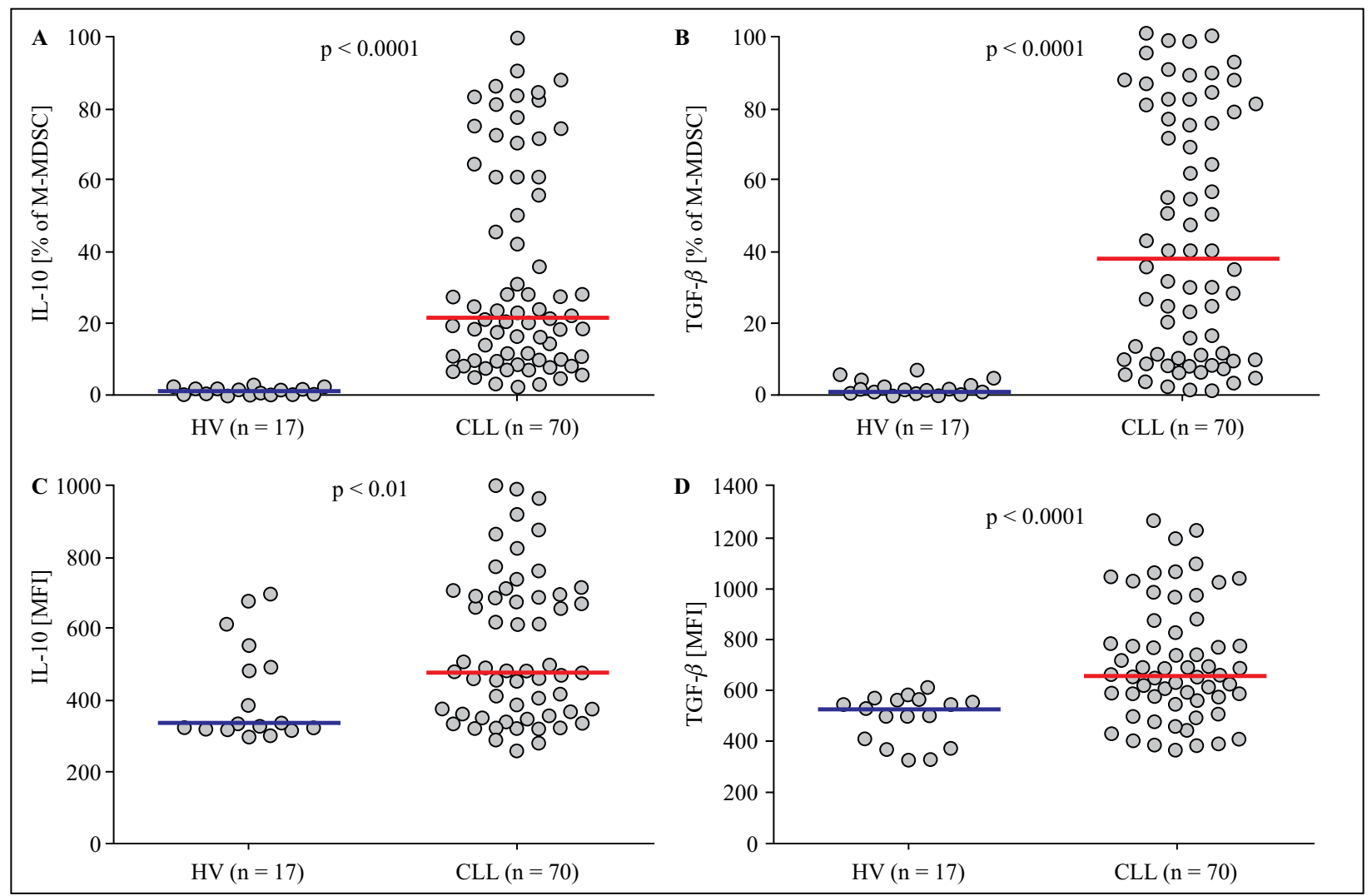

Figure 2. IL-10 and TGF- $\beta$ expression in M-MDSCs from CLL patients and healthy volunteers (HV). (A) percentage of IL-10 positive M-MDSCs; (B) mean fluorescence intensity (MFI) of IL-10 in M-MDSCs. (C) percentage of TGF- $\beta$ positive M-MDSCs; (D) MFI of TGF- $\beta$ in M-MDSCs. The U Mann-Whitney test was used for comparative analysis.

with intracellular IL-10 expression in CLL patients than in healthy volunteers (median [IQR], $21.72 \%$ [9.81-60.93\%] vs. $0.57 \%$ [0.06-1.31\%], p < 0.0001; Fig. 2A). Likewise, percentage of M-MDSC with TGF- $\beta 1$ (median [IQR], 37.36\% [9.93-78.37\%]) expression was significantly higher in comparison to the HVs (median [IQR], 1.17\% [0.33-3.68\%) (Fig. 2B). Furthermore, within M-MDSC population, IL-10 and TGF- $\beta 1$ cytokines were quantified regarding their mean fluorescence intensity (MFI). CLL patients increased IL-10 expression level (median [IQR], 477.0 [355.3-689.3] MFI), when compared to healthy controls (median [IQR], 333.0 [318.2-522.2] MFI) $(\mathrm{p}<0.01)$ (Fig. 2C). Likewise, TGF- $\beta 1$ expression level indicated by MFI was significantly higher in M-MDSC from CLL patients when compared to HV (median [IQR], 658.4 [563.6-865.2] MFI vs. 526.60 [389.6-565.8] MFI, $p<0.001$ ) (Fig. 2D).

The percentage of M-MDSC with intracellular IL-10 expression showed low inter-individual variability in healthy controls while in CLL patients it was much more diverse and significantly lower in patients at stage 0 as compared to the stages I/II and III/IV ac- cording to Rai stages (Fig. 3A, Table 2). Likewise, MFI was lower in patients at stage 0 (median, 456.0 MFI) as compared to the stages I/II (median, $481.90 \mathrm{MFI}$ ) and III/IV (median, 757.30 MFI) (Fig. 3B, Table 2). Similarly the percentage of M-MDSC with intracellular TGF- $\beta 1$ expression was significantly lower in patients at stage 0 as compared to the stages $\mathrm{I} / \mathrm{II}$ and III/IV according to Rai stages (Fig. 3C, Table 2). Likewise, MFI was lower in patients at stage 0 as compared to the stages I/II and III/IV (Fig. 3D, Table 2).

The percentage of M-MDSC with intracellular TGF- $\beta 1$ expression was significantly higher in ZAP-70-positive patients compared with ZAP-70-negative ones (Fig. 4A, Table 3) $(\mathrm{p}<0.05)$. Likewise, higher intracellular TGF- $\beta 1$ expression determined by MFI was observed in ZAP-70+ than in ZAP-70- patients $(\mathrm{p}<0.05)$ (Fig. 4B, Table 3). We also observed a higher percentage of M-MDSC with TGF- $\beta 1$ expression in CD38-negative patients than in CD38-positive ones $(p<0.05)$ (Fig. 4C, Table 3). Likewise, MFI was higher in CD38+ than in CD38patients; however, the difference was not statistically significant ( $p>0.05$ ) (Fig. 4D, Table 3). Opposing 


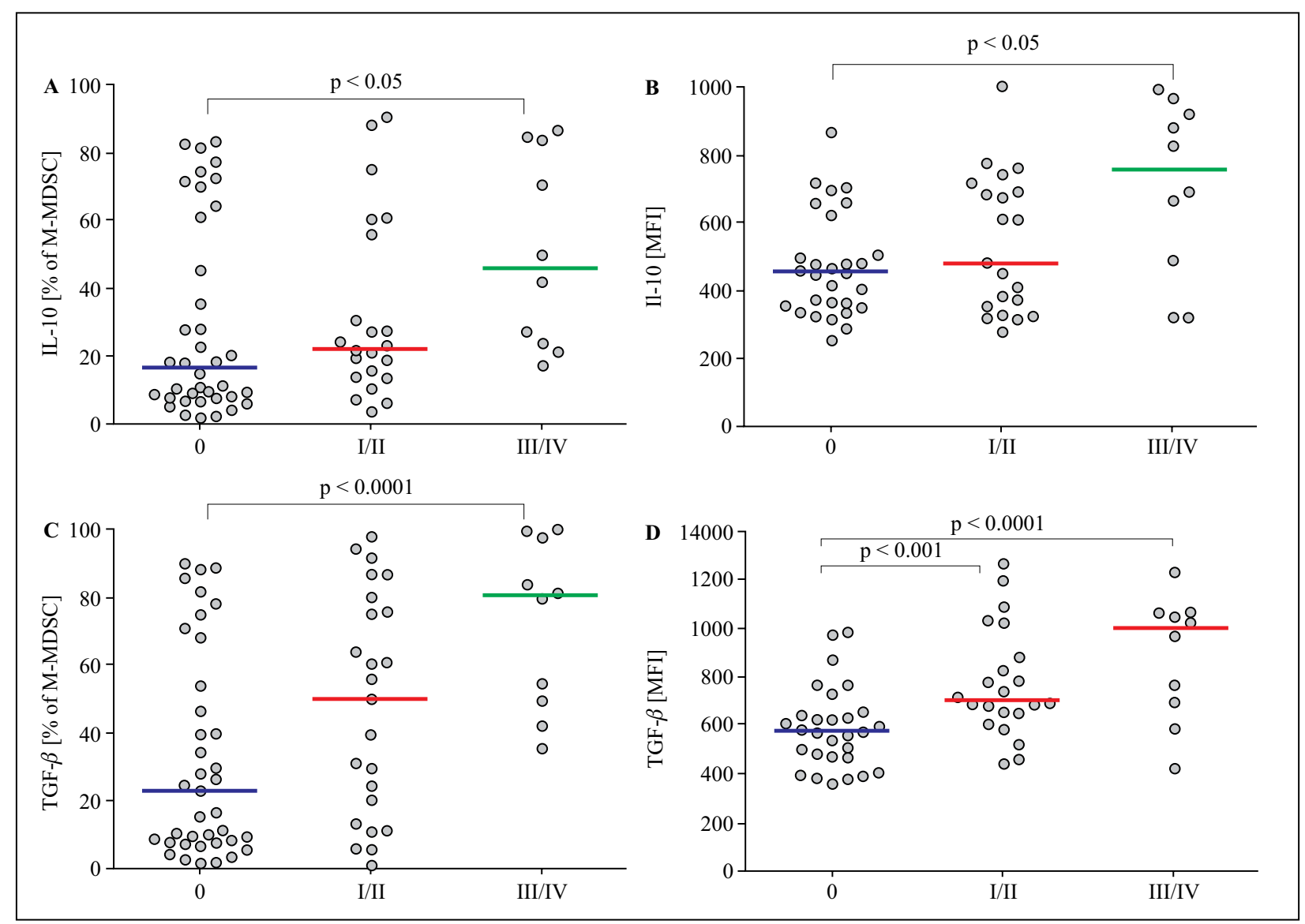

Figure 3. IL-10 and TGF- $\beta$ expression in M-MDSCs from CLL patients in different disease stages. (A) percentage of IL-10 positive M-MDSCs; (B) mean fluorescence intensity (MFI) of IL-10 in M-MDSCs. (C) percentage of TGF- $\beta$ positive M-MDSCs; (D) MFI of TGF- $\beta$ in M-MDSCs. The Kruskal-Wallis test with Dunn correction was used for comparison or comparative analysis.

Table 2. M-MDSC with intracellular IL-10 or TGF- $\beta 1$ expression in CLL patients at various disease stages (three risk groups)

\begin{tabular}{|c|c|c|c|c|c|c|}
\hline \multicolumn{7}{|c|}{ 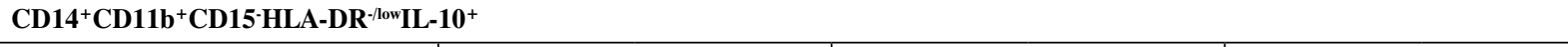 } \\
\hline & \multicolumn{2}{|c|}{ Stage $0(n=37)$} & \multicolumn{2}{|c|}{ Stage I/II $(n=23)$} & \multicolumn{2}{|c|}{ Stage III/IV (n = 10) } \\
\hline & $\%$ & MFI & $\%$ & MFI & $\%$ & MFI \\
\hline Median & 17.18 & 456.0 & 22.80 & 481.90 & 46.14 & 757.30 \\
\hline Minimum & 2.32 & 258.2 & 4.28 & 279.20 & 17.51 & 318.70 \\
\hline Maximum & 83.77 & 864.6 & 90.63 & 1000.00 & 86.40 & 991.50 \\
\hline IQR & 53.65 & 212.6 & 42.85 & 357.6 & 60.39 & 484.00 \\
\hline \multicolumn{7}{|c|}{ 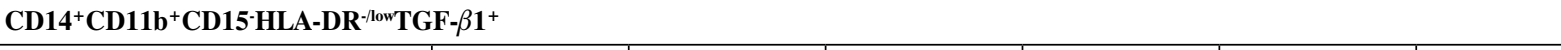 } \\
\hline & $\%$ & MFI & $\%$ & MFI & $\%$ & MFI \\
\hline Median & 22.89 & 582.70 & 50.10 & 704.90 & 80.43 & 1000.00 \\
\hline Minimum & 1.29 & 365.20 & 1.27 & 445.70 & 35.36 & 428.8 \\
\hline Maximum & 89.41 & 987.90 & 97.63 & 1269.0 & 99.48 & 1230.0 \\
\hline IQR & 53.01 & 175.60 & 66.76 & 261.70 & 49.69 & 393.40 \\
\hline
\end{tabular}

MFI - Mean fluorescence intensity 


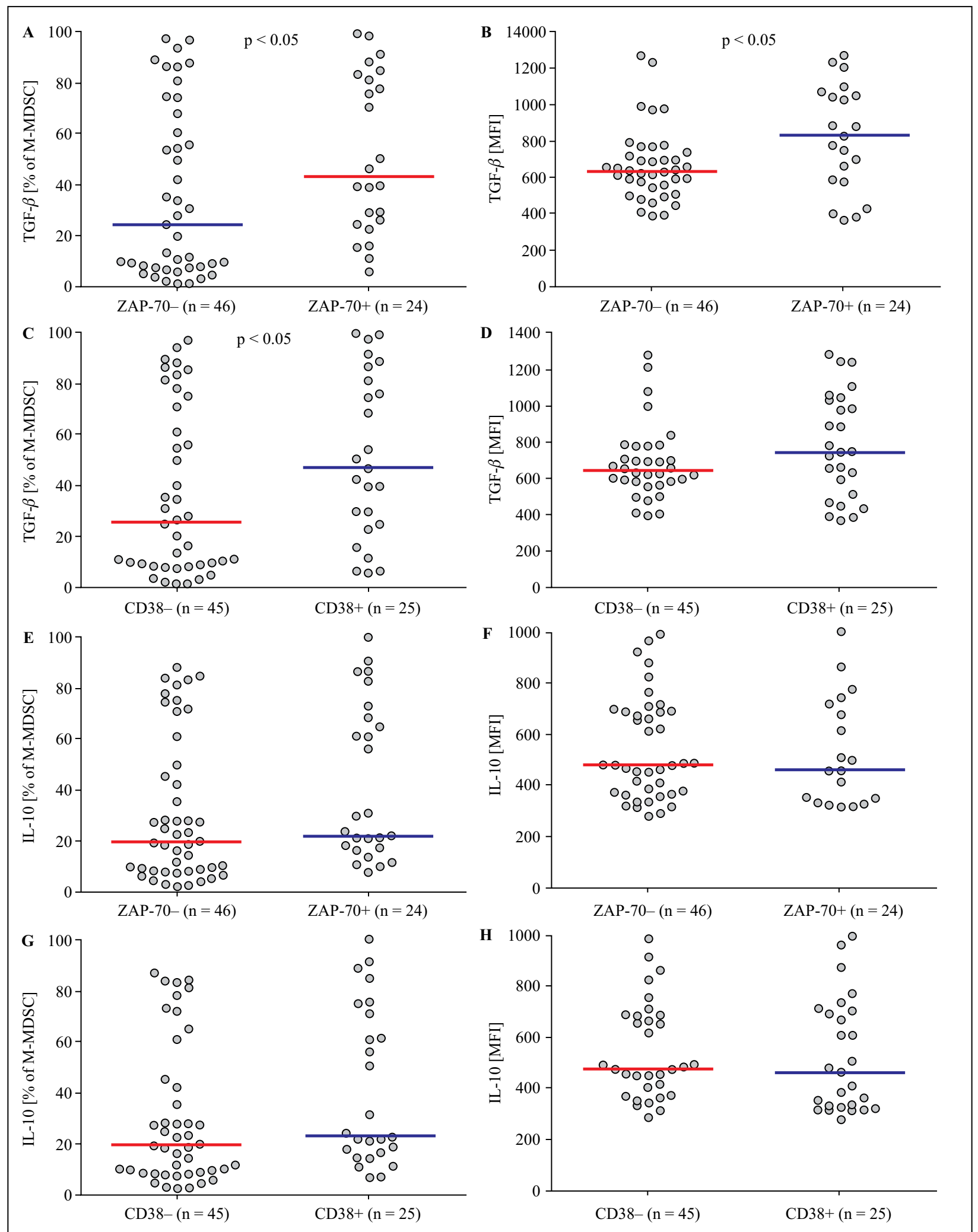

Figure 4. IL-10 and TGF- $\beta$ expression in M-MDSCs from CLL patients analyzed by adverse prognostic factors. A. The percentage of $\mathrm{CD} 14^{+} \mathrm{CD} 11 \mathrm{~b}^{+} \mathrm{CD} 15-\mathrm{HLA}-\mathrm{DR}{ }^{-/ 10 w}$ TGF- $\beta^{+}$cells in ZAP-70-negative patients compared with ZAP-70-positive patients. B. TGF- $\beta$ expression levels indicated by the MFI (mean fluorescence intensity) in M-MDSCs from ZAP-70-negative and ZAP-70-positive patients. C. The percentage of $\mathrm{CD}_{14}{ }^{+} \mathrm{CD} 11 \mathrm{~b}^{+} \mathrm{CD} 15$-HLA-DR-/ow TGF- $\beta^{+}$cells in CD38-negative patients compared with CD38-positive patients. D. TGF- $\beta$ expression levels indicated by the MFI in M-MDSCs from CD38- and CD38+ patients. E. The percentage of CD14 ${ }^{+}$CD $11 b^{+}$CD15-HLA$\mathrm{DR}^{-1 / \mathrm{low}} \mathrm{IL}-10^{+}$cells in ZAP-70-negative patients compared with ZAP-70-positive patients. F. IL-10 expression levels indicated by the MFI in M-MDSCs from ZAP-70-negative and ZAP-70-positive patients. G. The percentage of CD14 ${ }^{+} \mathrm{CD} 11 \mathrm{~b}^{+} \mathrm{CD} 15-\mathrm{HLA}-\mathrm{DR}{ }^{-/ / \text {low }} \mathrm{IL}-10^{+}$cells in CD38-negative patients compared with CD38-positive patients. H. IL-10 expression levels indicated by the MFI in M-MDSCs from CD38and CD38+ patients. The U Mann-Whitney test was used for comparative analysis. 
Table 3. Percentage of M-MDSC with the expression of IL-10 and IL-10 MFI in CLL patients classified into groups: ZAP-70+, ZAP-70-, CD38+, CD38-

\begin{tabular}{|c|c|c|c|c|c|c|c|c|}
\hline \multicolumn{9}{|c|}{ M-MDSC IL10-positive } \\
\hline & \multicolumn{2}{|c|}{$\mathrm{ZAP}-70+(\mathrm{n}=24)$} & \multicolumn{2}{|c|}{ ZAP-70- $(n=46)$} & \multicolumn{2}{|c|}{ CD38+ $(n=25)$} & \multicolumn{2}{|c|}{ CD38- $(n=45)$} \\
\hline & $\%$ & MFI & $\%$ & MFI & $\%$ & MFI & $\%$ & MFI \\
\hline Median & 21.95 & 458.20 & 20.02 & 478.00 & 22.91 & 465.60 & 19.67 & 478.00 \\
\hline Minimum & 7.70 & 316.60 & 2.32 & 279.20 & 6.640 & 279.20 & 2.320 & 290.70 \\
\hline Maximum & 99.84 & 1000.00 & 88.24 & 991.50 & 99.84 & 1000.00 & 86.40 & 991.50 \\
\hline IQR & 48.33 & 382.90 & 41.80 & 318.70 & 55.59 & 380.50 & 36.32 & 296.50 \\
\hline \multicolumn{9}{|c|}{ M-MDSC TGF- $\beta$ 1-positive } \\
\hline & $\%$ & MFI & $\%$ & MFI & $\%$ & MFI & $\%$ & MFI \\
\hline Median & 43.28 & 830.20 & 24.56 & 631.80 & 25.51 & 738.70 & 46.71 & 639.40 \\
\hline Minimum & 6.18 & 365.20 & 1.27 & 387.70 & 1.27 & 365.20 & 5.57 & 392.90 \\
\hline Maximum & 99.48 & 1269.00 & 97.63 & 1265.0 & 97.14 & 1269.00 & 99.48 & 1265.0 \\
\hline IQR & 57.85 & 476.80 & 60.33 & 189.90 & 60.20 & 532.20 & 63.41 & 197.20 \\
\hline
\end{tabular}
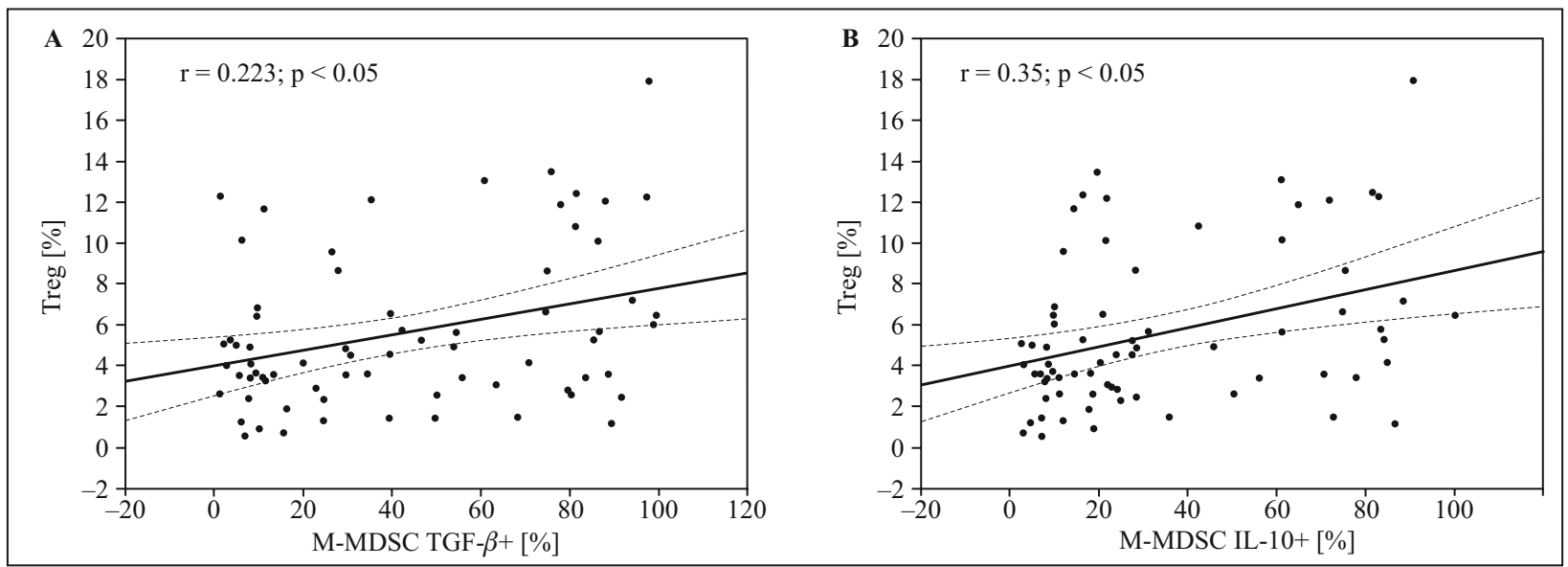

Figure 5. The relationship between the percentages of Treg lymphocytes and M-MDSCs with intracellular TGF- $\beta$ (A) or IL-10 (B) expression. The Spearman rank correlation coefficient was used in correlation tests.

to what was observed for TGF- $\beta 1$ differences in percentage of M-MDSC with IL-10 expression and IL-10 MFI levels were not statistically significant between ZAP-70+ and ZAP-70- patients (Fig. 4E, $4 \mathrm{~F})$. Likewise, the difference between CD38-negative patients and CD38-positive ones was not statistically significant ( $p>0.05$ ) (Fig. 4G, 4H).

The percentage of M-MDSC with TGF- $\beta 1$ expression correlated positively with the peripheral blood WBC count $(\mathrm{r}=0.272 ; \mathrm{p}<0.05)$ and lymphocyte count $(r=0.260 ; p<0.05)$. In the group of patients with CLL, no significant correlations were identified between the frequency of M-MDSC TGF- $\beta 1$-positive and platelet counts, hemoglobin concentration, serum LDH and $\beta 2$-microglobulin levels or the age of the patients.
There was a significant correlation between the percentage of M-MDSC with intracellular TGF- $\beta 1$ expression and Treg cells $(r=0.223 ; p<0.05)$ (Fig. 5A). Likewise, percentage of M-MDSC cell with IL-10 expression correlated with frequency of Treg $(r=0.35$; $\mathrm{p}<0.05)$ (Fig. 5B).

Moreover, there was a significant difference in the median percentage of M-MDSC with intracellular IL-10 expression between patients carrying the $11 \mathrm{q} 22.3$ and/or the 17p13.1 deletion (median [IQR], 31.41\% [21.29-76.96\%]) and patients without these genetic aberrations (median [IQR], 20.67\% [10.80-58.36\%]) $(\mathrm{p}<0.05)$ (Fig. 6A). We also observed a significantly higher percentage of M-MDSC positive for intracellular TGF- $\beta$ expression in patients carrying the 11q22.3 and/or the 17p13.1 deletion (median [IQR], 55.10\% 


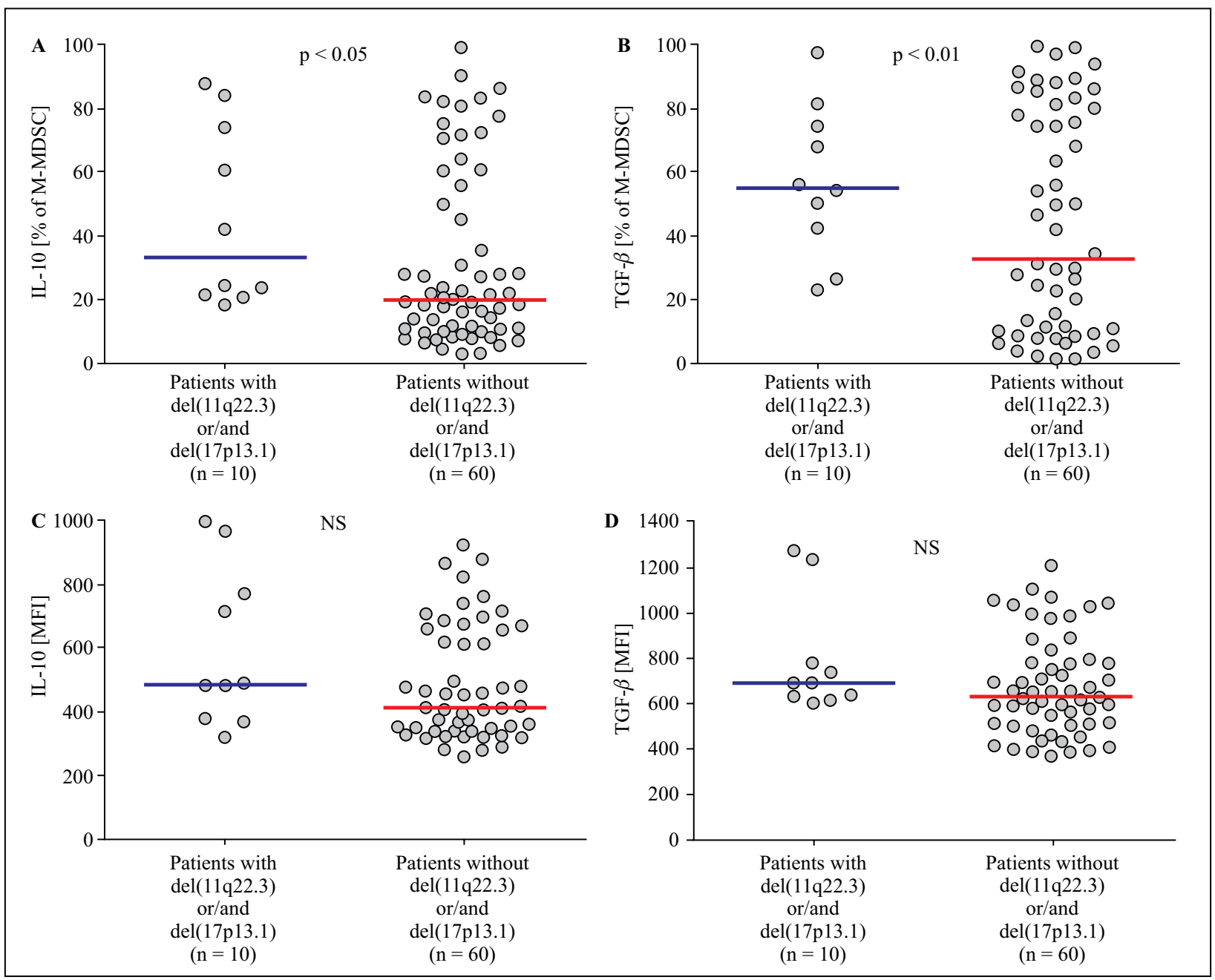

Figure 6. IL-10 and TGF- $\beta$ expression in M-MDSCs from CLL patients carrying the $11 \mathrm{q} 22.3$ and/or the $17 \mathrm{p} 13.1$ deletion and patients without these genetic aberrations. (A) percentage of IL-10 positive M-MDSCs; (B) percentage of TGF- $\beta$ positive M-MDSCs; (C) mean fluorescence intensity (MFI) of IL-10 in M-MDSCs; (D) MFI of TGF- $\beta$ in M-MDSCs. The U Mann-Whitney test was used for comparative analysis.

[38.30-76.18\%]) than in patients without these genetic aberrations (median [IQR], 32.60\% [9.82-80.53\%] $(\mathrm{p}<0.01)$ (Fig. 6B).

Likewise, TGF- $\beta 1$ and IL-10 expression levels indicated by the MFI were compared between patients carrying the $11 \mathrm{q} 22.3$ and/or the $17 \mathrm{p} 13.1$ deletion (TGF- $\beta$ 1: median [IQR], 674.4 [571.5-903.0] MFI; IL-10: median [IQR], 470.8 [366.2-822.8] MFI) and patients without these genetic aberrations (TGF- $\beta 1$ : median [IQR], 629.5 [495.5-808.0] MFI; IL-10: median [IQR], 411.8 [334.2-656.7] MFI). However, the difference was not statistically significant $(p>0.05)$ (Fig. 6C, 6D).

During the follow-up period (range 1-55 months), 17 patients required therapy (Table 1 ). The percentage of M-MDSC IL-10-positive and M-MDSC TGF- $\beta 1$-positive measured at the time of diagnosis was statistically higher in patients requiring therapy as compared to patients without treatment during the observation period (IL-10: median [IQR], 27.95\% [11.73-99.84\%] vs. $21.48 \%$ [18.08-49.01\%] TGF- $\beta 1$ : median [IQR], 49.76\% [24.56-83.46\%] vs. $39.35 \%$ [13.77-77.72\%] $(\mathrm{p}<0.05)$. However, MFI level was not statistically significant between both groups (IL-10: median [IQR], 477.0 [372.2-696.0] MFI vs. 375.3 [319.3-774.9] MFI; TGF- $\beta 1$ : median [IQR], 735.7 [477.1-927.3] MFI vs. 651.5 [575.5-876.9] MFI; $\mathrm{p}>0.05)$.

\section{Discussion}

The presence of M-MDSC in the microenvironment has recently aroused great interest. M-MDSC, like regulatory $\mathrm{T}$ cells, is thought to contribute to the 
effective reduction of anti-tumor response [30]. Unfortunately, there are very few studies available on M-MDSCs in CLL patients [16, 31-33]. Jitschin et al. [16] identified increased numbers of MDSCs $\left(\mathrm{CD} 14^{+}\right.$HLA-DR $\left.{ }^{\mathrm{lo}}\right)$ in untreated patients with CLL. This finding is supported by our results showing the M-MDSC (defined as CD14 ${ }^{+} \mathrm{CD} 11 \mathrm{~b}^{+} \mathrm{CD} 15$-HLA$-\mathrm{DR}^{- \text {llow }}$ cells) accumulation in CLL patients.

M-MDSCs have been described to express IDO (indoleamine-2,3-dioxygenase). IDO activity is an important mechanism by which effector $T$ cells can be induced and converted to Tregs. The IDO-mediated depletion of tryptophan and subsequent production of immunosuppressive products may also lead to $\mathrm{T}$ cell suppression through the down-regulation of the TCR-CD3- $\zeta$ chain [34]. IDO ${ }^{\text {high }}$ MDSCs suppress both T-cell activation and proliferation in untreated CLL patients [16]. M-MDSCs have also been described to secrete TGF- $\beta$ and IL-10, which exert direct immunosuppressive effects on $\mathrm{T}$ effector cells or induce the generation of Treg cells [35].

Alhakeem et al. [19] observed that CLL patients have a significantly higher level of IL-10 in plasma than healthy donors. They reported that IL-10 affects CLL growth indirectly by suppressing anti-CLL T-cells. In their study IL-10 reduced the generation of effector CD4 and CD8 T-cells [19]. Still little is known about the mechanisms that lead to the increasing of M-MDSC, especially in the microenvironment of leukemia [20] and lymphoma [24]. Lee et al. [36] found that TGF- $\beta$ induced the expansion of the monocytic MDSC population, expression of immunosuppressive molecules by MDSCs, and the ability of MDSCs to suppress CD4+ T cell proliferation. In our study, we found a significantly higher percentage of M-MDSC with intracellular IL-10 or TGF- $\beta$ expression in CLL patients than in healthy volunteers. Moreover, we reported a significant correlation between the percentage of M-MDSC with TGF- $\beta$ or IL-10 expression and the frequency of Treg cells. In general, the accumulation of Treg lymphocytes in CLL is well documented. Their increased incidence in patients with CLL affects the severity of the disease and worse prognosis [37].

Recently, Zahran AM et al. [33] reported that the abundance of $\mathrm{CD}_{14}{ }^{+} \mathrm{HLA}-\mathrm{DR}{ }^{\mathrm{lo}}$ cells is associated with frequency of ZAP-70 and CD38 cells. Expression of CD38 and ZAP-70 in cancer cells of CLL patients are typical immunophenotypic unfavorable prognostic markers of patients' life expectancy [38]. It is interesting that M-MDSC from ZAP-70-positive and CD38-positive cases expressed higher TGF- $\beta$ or IL-10 levels. What is more, we were able to demonstrate a significant association of TGF or IL-10 expression in M-MDSC with del(11q22.3) and/or del(17p13.1). Deletions in the long arm of chromosomes 11 [del(11q)] or in the short arm of chromosome 17 [del(17p)] are recognized as high-risk genetic lesions [26]. Del(17p) is associated with an aggressive clinical course. Patients with leukemia cells that have del(17p) are resistant to standard chemotherapy regimens containing alkylating agents and purine analogs [26, $39,40]$. Likewise, the presence of $\operatorname{del}(11 q)$ is associated with a clinically progressive disease in almost all CLL cases [41]. Patients with leukemia cells that have del(11q) have been associated with shorter TTFT (time to first treatment), shorter remission durations, and shorter OS (overall survival) following standard chemotherapy compared to cases without this genetic aberration [41].

Gustafson et al. [31] reported that the abundance of CD14 ${ }^{+} \mathrm{HLA}-\mathrm{DR}{ }^{\mathrm{lo}}$ cells is associated with a shorter time to CLL progression. Likewise, Liu et al. [32] reported that upregulation of CD $14^{+} \mathrm{HLA}-\mathrm{DR}{ }^{\mathrm{low} /}$ MDSCs correlated with CLL progression and a poor prognosis for CLL patients. Recently, Zahran AM et al. [33] reported that the survival time was significantly shorter in patients expressed high levels of M-MDSC when compared to those with low levels. In our study patients who required therapy had a higher percentage of M-MDSC with TGF- $\beta$ or IL-10 expression. Moreover, the percentage of CD14 ${ }^{+}$HLA-DR-llow cells with TGF- $\beta$ or IL-10 expression increased with more advanced clinical stage of CLL. Undoubtedly, the existence of a relationship between the number of CD14+HLA-DR ${ }^{- \text {llow }}$ cells and the stage of clinical advancement of the disease indicates the possible involvement of CD14 ${ }^{+} \mathrm{HLA}^{-\mathrm{DR}^{-/ \text {/low }}}$ cells in CLL progression. According to the data presented by Jitschin et al. [16], patients with a higher M-MDSC percentage have a more rapid accumulation of leukemia lymphocytes. In our study, the percentage of M-MDSC with TGF- $\beta$ expression correlated positively with the WBC count and PB lymphocyte count.

This is the first report investigated the potential role of M-MDSCs in CLL by analyzing the level of IL-10 and TGF- $\beta 1$ expression in circulating M-MDSCs in correlation with clinical and laboratory parameters characterizing disease activity and patients' immune status. We have shown that an increased percentage of M-MDSC cells producing IL- 10 and TGF- $\beta 1$ patients may be associated with suppression of the immune response against leukemic cells. It can be assumed that the increased percentage of M-MDSC with intracellular expression of IL-10 and TGF- $\beta 1$ may in the future be the factor defining the group of patients with shorter time to onset of treatment. 


\section{Acknowledgments}

This work was supported by a grant from the Medical University of Lublin (grant no. DS 458).

\section{Authors' contributions}

$\mathrm{ABJ}$ - designed research, performed research, analyzed and interpreted data, wrote the paper, critically revised the manuscript. WK - performed research, collected medical data, wrote the paper and critically revised the manuscript.

\section{Conflict of interest}

The authors declare that they have no conflict of interest.

\section{References}

1. Mohr A, Renaudineau Y, Bagacean C, et al. Regulatory B lymphocyte functions should be considered in chronic lymphocytic leukemia. Oncoimmunology. 2016; 5(5): e1132977, doi: 10.1080/2162402X.2015.1132977, indexed in Pubmed: 27467951.

2. Ten Hacken E, Burger JA. Microenvironment interactions and B-cell receptor signaling in Chronic Lymphocytic Leukemia: Implications for disease pathogenesis and treatment. Biochim Biophys Acta. 2016; 1863(3): 401-413, doi: 10.1016/j. bbamcr.2015.07.009, indexed in Pubmed: 26193078.

3. ten Hacken E, Burger JA. Microenvironment dependency in Chronic Lymphocytic Leukemia: The basis for new targeted therapies. Pharmacol Ther. 2014; 144(3): 338-348, doi: 10.1016/j.pharmthera.2014.07.003, indexed in Pubmed: 25050922.

4. van Attekum MHa, Eldering E, Kater AP. Chronic lymphocytic leukemia cells are active participants in microenvironmental cross-talk. Haematologica. 2017; 102(9): 1469-1476, doi: 10.3324/haematol.2016.142679, indexed in Pubmed: 28775118.

5. Ostrand-Rosenberg S, Fenselau C. Myeloid-Derived Suppressor Cells: Immune-Suppressive Cells That Impair Antitumor Immunity and Are Sculpted by Their Environment. J Immunol. 2018; 200(2): 422-431, doi: 10.4049/jimmunol.1701019, indexed in Pubmed: 29311384.

6. Gabrilovich DI, Nagaraj S. Myeloid-derived suppressor cells as regulators of the immune system. Nat Rev Immunol. 2009; 9(3): 162-174, doi: 10.1038/nri2506, indexed in Pubmed: 19197294.

7. Nicholas NS, Apollonio B, Ramsay AG. Tumor microenvironment (TME)-driven immune suppression in B cell malignancy. Biochim Biophys Acta. 2016; 1863(3): 471-482, doi: 10.1016/j.bbamcr.2015.11.003, indexed in Pubmed: 26554850.

8. Palumbo GA, Parrinello NL, Giallongo C, et al. Monocytic myeloid derived suppressor cells in hematological malignancies. Int J Mol Sci. 2019; 20(21), doi: 10.3390/ijms20215459, indexed in Pubmed: 31683978.

9. Giallongo C, Parrinello N, Brundo M, et al. Myeloid derived suppressor cells in chronic myeloid leukemia. Frontiers in Oncology. 2015; 5, doi: 10.3389/fonc.2015.00107.

10. Giallongo C, Parrinello NL, La Cava P, et al. Monocytic myeloid-derived suppressor cells as prognostic factor in chronic myeloid leukaemia patients treated with dasatinib. J Cell
Mol Med. 2018; 22(2): 1070-1080, doi: 10.1111/jcmm.13326, indexed in Pubmed: 29218828.

11. Marvel D, Gabrilovich DI. Myeloid-derived suppressor cells in the tumor microenvironment: expect the unexpected. J Clin Invest. 2015; 125(9): 3356-3364, doi: 10.1172/JCI80005, indexed in Pubmed: 26168215.

12. Bronte V, Brandau S, Chen SH, et al. Recommendations for myeloid-derived suppressor cell nomenclature and characterization standards. Nat Commun. 2016; 7: 12150, doi: 10.1038/ ncomms12150, indexed in Pubmed: 27381735.

13. Wang Y, Ding Y, Guo N, et al. MDSCs: Key Criminals of Tumor Pre-metastatic Niche Formation. Front Immunol. 2019; 10: 172, doi: 10.3389/fimmu.2019.00172, indexed in Pubmed: 30792719.

14. Höpken UE, Rehm A. Targeting the Tumor Microenvironment of Leukemia and Lymphoma. Trends Cancer. 2019; 5(6): 351-364, doi: 10.1016/j.trecan.2019.05.001, indexed in Pubmed: 31208697.

15. Zirlik K. MDSCs: the final frontier of the microenvironment in CLL? Blood. 2014; 124(5): 666-668, doi: 10.1182/ blood-2014-06-578880, indexed in Pubmed: 25082859.

16. Jitschin R, Braun M, Büttner M, et al. CLL-cells induce IDOhi CD14+HLA-DRlo myeloid-derived suppressor cells that inhibit T-cell responses and promote TRegs. Blood. 2014; 124(5): 750-760, doi: 10.1182/blood-2013-12-546416, indexed in Pubmed: 24850760.

17. Özkan B, Lim H, Park SG. Immunomodulatory function of myeloid-derived suppressor cells during B cell-mediated immune responses. Int J Mol Sci. 2018; 19(5), doi: 10.3390/ ijms19051468, indexed in Pubmed: 29762501.

18. Tcyganov E, Mastio J, Chen E, et al. Plasticity of myeloid-derived suppressor cells in cancer. Curr Opin Immunol. 2018; 51: 76-82, doi: 10.1016/j.coi.2018.03.009, indexed in Pubmed: 29547768.

19. Alhakeem SS, McKenna MK, Oben KZ, et al. Chronic lymphocytic leukemia-derived IL-10 suppresses antitumor immunity. J Immunol. 2018; 200(12): 4180-4189, doi: 10.4049/ jimmunol.1800241, indexed in Pubmed: 29712773.

20. Lv M, Wang Ke, Huang XJ. Myeloid-derived suppressor cells in hematological malignancies: friends or foes. J Hematol Oncol. 2019; 12(1): 105, doi: 10.1186/s13045-019-0797-3, indexed in Pubmed: 31640764.

21. Millrud CR, Bergenfelz C, Leandersson K. On the origin of myeloid-derived suppressor cells. Oncotarget. 2017; 8(2): 3649-3665, doi: 10.18632/oncotarget.12278, indexed in Pubmed: 27690299.

22. Kumar V, Patel S, Tcyganov E, et al. The nature of myeloid-derived suppressor cells in the tumor microenvironment. Trends Immunol. 2016; 37(3): 208-220, doi: 10.1016/j. it.2016.01.004, indexed in Pubmed: 26858199.

23. Bergenfelz C, Larsson AM, von Stedingk K, et al. Systemic monocytic-MDSCs are generated from monocytes and correlate with disease progression in breast cancer patients. PLoS One. 2015; 10(5): e0127028, doi: 10.1371/journal. pone.0127028, indexed in Pubmed: 25992611.

24. Xiu B, Lin Y, Grote DM, et al. IL-10 induces the development of immunosuppressive CD14(+)HLA-DR(low/-) monocytes in B-cell non-Hodgkin lymphoma. Blood Cancer J. 2015; 5: e328, doi: 10.1038/bcj.2015.56, indexed in Pubmed: 26230952.

25. Kowalska W. Expression of CD163 and HLA-DR molecules on the monocytes in chronic lymphocytic leukemia patients. Folia Histochem Cytobiol. 2020 [Epub ahead of print], doi: 10.5603/FHC.a2020.0002, indexed in Pubmed: 32176313.

26. Hallek M, Cheson BD, Catovsky D, et al. International workshop on chronic lymphocytic leukemia. Guidelines for the 
diagnosis and treatment of chronic lymphocytic leukemia: a report from the International Workshop on Chronic Lymphocytic Leukemia updating the National Cancer Institute-Working Group 1996 guidelines. Blood. 2008; 111(12): 5446-5456, doi: 10.1182/blood-2007-06-093906, indexed in Pubmed: 18216293.

27. Rai KR, Sawitsky A, Cronkite EP, et al. Clinical staging of chronic lymphocytic leukemia. Blood. 1975; 46(2): 219-234, doi: 10.1182/blood.v46.2.219.219.

28. Hus I, Podhorecka M, Bojarska-Junak A, et al. The clinical significance of ZAP-70 and CD38 expression in B-cell chronic lymphocytic leukaemia. Ann Oncol. 2006; 17(4): 683-690, doi: 10.1093/annonc/mdj120, indexed in Pubmed: 16524977.

29. Bojarska-Junak A, Hus I, Chocholska S, et al. CD1d expression is higher in chronic lymphocytic leukemia patients with unfavorable prognosis. Leuk Res. 2014; 38(4): 435-442, doi: 10.1016/j.leukres.2013.12.015, indexed in Pubmed: 24418751.

30. Awad RM, De Vlaeminck Y, Maebe J, et al. Turn back the TIMe: targeting tumor infiltrating myeloid cells to revert cancer progression. Front Immunol. 2018; 9: 1977, doi: 10.3389/ fimmu.2018.01977, indexed in Pubmed: 30233579.

31. Gustafson MP, Abraham RS, Lin Yi, et al. Association of an increased frequency of CD14+ HLA-DR lo/neg monocytes with decreased time to progression in chronic lymphocytic leukaemia (CLL). Br J Haematol. 2012; 156(5): 674-676, doi: 10.1111/j.1365-2141.2011.08902.x, indexed in Pubmed: 22050346.

32. Liu J, Zhou Y, Huang Q, et al. CD14HLA-DR expression: A novel prognostic factor in chronic lymphocytic leukemia. Oncol Lett. 2015; 9(3): 1167-1172, doi: 10.3892/ol.2014.2808, indexed in Pubmed: 25663875.

33. Zahran AM, Moeen SM, Thabet AF, et al. Monocytic myeloid-derived suppressor cells in chronic lymphocytic leukemia patients: a single center experience. Leuk Lymphoma. 2020 [Epub ahead of print]: 1-8, doi: 10.1080/10428194.2020.1728747, indexed in Pubmed: 32077360 .
34. Li F, Zhao Y, Wei L, et al. Tumor-infiltrating Treg, MDSC, and IDO expression associated with outcomes of neoadjuvant chemotherapy of breast cancer. Cancer Biol Ther. 2018; 19(8): 695-705, doi: 10.1080/15384047.2018.1450116, indexed in Pubmed: 29621426.

35. Groth $\mathrm{C}, \mathrm{Hu} \mathrm{X}$, Weber $\mathrm{R}$, et al. Immunosuppression mediated by myeloid-derived suppressor cells (MDSCs) during tumour progression. Br J Cancer. 2019; 120(1): 16-25, doi: 10.1038/ s41416-018-0333-1, indexed in Pubmed: 30413826.

36. Lee CR, Lee W, Cho S, et al. Characterization of multiple cytokine combinations and TGF- $\beta$ on differentiation and functions of myeloid-derived suppressor cells. IJMS. 2018; 19(3): 869, doi: 10.3390/ijms19030869.

37. Lad DP, Varma S, Varma N, et al. Regulatory T-cell and T-helper 17 balance in chronic lymphocytic leukemia progression and autoimmune cytopenias. Leuk Lymphoma. 2015; 56(8): 2424-2428, doi: 10.3109/10428194.2014.986479, indexed in Pubmed: 25393804.

38. Rassenti LZ, Jain S, Keating MJ, et al. Relative value of ZAP$70, \mathrm{CD} 38$, and immunoglobulin mutation status in predicting aggressive disease in chronic lymphocytic leukemia. Blood. 2008; 112(5): 1923-1930, doi: 10.1182/blood-2007-05-092882, indexed in Pubmed: 18577710.

39. Shindiapina P, Brown JR, Danilov AV. A new hope: novel therapeutic approaches to treatment of chronic lymphocytic leukaemia with defects in TP53. Br J Haematol. 2014; 167(2): 149-161, doi: 10.1111/bjh.13042, indexed in Pubmed: 25040077.

40. Brander D, Islam P, Barrientos JC. Tailored Treatment Strategies for Chronic Lymphocytic Leukemia in a Rapidly Changing Era. Am Soc Clin Oncol Educ Book. 2019; 39: 487-498, doi: 10.1200/EDBK_238735, indexed in Pubmed: 31099686.

41. Puiggros A, Blanco G, Espinet B. Genetic abnormalities in chronic lymphocytic leukemia: where we are and where we go. Biomed Res Int. 2014; 2014: 435983, doi: 10.1155/2014/435983, indexed in Pubmed: 24967369.

Submitted: 28 February, 2020

Accepted after reviews: 24 March, 2020 Available as AoP: 30 March, 2020 